\title{
DIAGONALIZATION IN COMPACT LIE ALGEBRAS AND A NEW PROOF OF A THEOREM OF KOSTANT
}

\author{
N. J. WILDBERGER
}

(Communicated by Jonathan M. Rosenberg)

\begin{abstract}
We exhibit a simple algorithmic procedure to show that any element of a compact Lie algebra is conjugate to an element of a fixed maximal abelian subalgebra. An estimate of the convergence of the algorithm is obtained. As an application, we provide a new proof of Kostant's theorem on the projection of orbits onto a maximal abelian subalgebra.
\end{abstract}

Let $M \in M(n, \mathbb{C})$ be a Hermitian matrix and consider the problem of diagonalizing $M$, that is, finding a unitary $n \times n$ matrix $g$ such that $g^{-1} M g$ is diagonal. This problem is essentially equivalent to that of finding the eigenvalues and eigenvectors of $M$. We propose an algorithm for solving this problem which utilizes the Lie algebra structure of $\mathfrak{g}$, the $n \times n$ skew-Hermitian matrices, and the adjoint action of $G$, the $n \times n$ unitary group, on $\mathfrak{g}$. In fact our method applies generally to any compact connected Lie group $G$ and its Lie algebra $\mathfrak{g}$.

Fix a maximal torus $T \subseteq G$ with Lie algebra $\mathfrak{t} \subseteq \mathfrak{g}$ and let

$$
\mathfrak{g}=\mathfrak{t} \oplus \sum_{\alpha \in \Sigma^{+}} \mathfrak{g}_{\alpha}
$$

be the decomposition of $\mathfrak{g}$ into weight spaces under the adjoint action of $T$. Here $\Sigma^{+}$is a set of positive roots and each space $\mathfrak{g}_{\alpha}$ is two-dimensional. Given $Z \in \mathfrak{g}$, we will write

$$
Z=Z_{0}+\sum_{\alpha \in \Sigma^{+}} Z_{\alpha}
$$

corresponding to $(0.1)$. The idea is then to choose $\alpha \in \Sigma^{+}$such that $Z_{\alpha}$ has maximum norm and then find $g \in G$ such that $\operatorname{Ad}(g) Z$ has no $\mathfrak{g}_{\alpha}$ component. This turns out to be essentially a problem in $\mathrm{SU}(2)$, which we can solve using only quadratic equations. If $d(Z)$ denotes the distance from $Z$ to the subspace

Received by the editors November 29, 1990 and, in revised form, February 25, 1992.

1991 Mathematics Subject Classification. Primary 22E15; Secondary 58F05.

Key words and phrases. Diagonalization, compact Lie algebra, Kostant's theorem. 
$\mathfrak{t}$, then we prove the inequality

$$
d(\operatorname{Ad}(g) Z) \leq \sqrt{(l-1) / l} d(Z)
$$

where $l$ is the number of positive roots.

We then repeat the procedure, getting a sequence $Z=Z^{1}, Z^{2}, \ldots$ converging to a point $Z^{\infty} \in \mathfrak{t}$. Then $Z^{\infty}$ is the diagonalized form of $Z \in \mathfrak{g}$. The rate of convergence is controlled by $(0.3)$.

As an application, we use the algorithm to provide a direct proof of a theorem of Kostant [1] which states that if $p: \mathfrak{g} \rightarrow \mathfrak{t}$ is the orthogonal projection and $O_{X}$ denotes the $\operatorname{Ad}(G)$ orbit through $X \in \mathfrak{t}$, then

$$
p\left(O_{X}\right)=\operatorname{conv}\left\{O_{X} \cap \mathfrak{t}\right\}
$$

where conv denotes convex hull. It is well known that $O_{X} \cap \mathfrak{t}$ is a finite set of points, the Weyl group orbit of $X$, so $p\left(O_{X}\right)$ is a convex polytope. Most existing proofs of Kostant's theorem (for example, the approaches of Atiyah [1, 2], Heckman [4], or Guillemin and Sternberg [3] using symplectic geometry) utilize Morse theory. Our proof is direct and conceptually simple.

The problem of diagonalization for $G=\mathrm{SU}(2)$ is easy. The group $G$ consists of all matrices of the form

$$
g=\left|\begin{array}{cc}
\alpha & \beta \\
-\bar{\beta} & \bar{\alpha}
\end{array}\right|
$$

where $\alpha, \beta \in \mathbb{C}$ satisfy $|\alpha|^{2}+|\beta|^{2}=1$. The Lie algebra $\mathfrak{g}=\operatorname{su}(2)$ consists of all matrices of the form

$$
X=\left|\begin{array}{cc}
i x & z \\
-\bar{z} & -i x
\end{array}\right|
$$

where $x \in \mathbb{R}$ and $z \in \mathbb{C}$, and $\mathfrak{t}$ consists of the one-dimensional diagonal subalgebra. The adjoint action is given by conjugation, so that for $g$ and $X$ as in (1.1) and (1.2),

$$
\operatorname{Ad}\left(g^{-1}\right) X=g^{-1} X g
$$

Given $X \in \mathfrak{g}$, the diagonalization problem is to find $g \in G$ such that $g^{-1} X g$ is diagonal. We may assume $z \neq 0$. The eigenvalues of $X$ are $i \lambda$ and $-i \lambda$ where $\lambda=\left(x^{2}+|z|^{2}\right)^{1 / 2}$ and the corresponding eigenvectors are

$$
\left|\begin{array}{c}
z \\
i(\lambda-x)
\end{array}\right| \text { and }\left|\begin{array}{c}
i(\lambda-x) \\
-\bar{z}
\end{array}\right|
$$

Both these vectors have length $d=\sqrt{2 \lambda(\lambda-x)}$. It follows that if we set

$$
g=\frac{1}{d}\left|\begin{array}{cc}
z & i(\lambda-x) \\
i(\lambda-x) & \bar{z}
\end{array}\right|
$$

then $g \in \mathrm{SU}(2)$ and

$$
g^{-1} X g=\left|\begin{array}{cc}
i \lambda & 0 \\
0 & -i \lambda
\end{array}\right|
$$

Note that we have used only quadratic equations to obtain $g$. 
Let $G$ be a compact, connected, semisimple Lie group with Lie algebra $\mathfrak{g}$. Let $($,$) be a G$-invariant positive-definite form on $\mathfrak{g}$ and || the associated norm. Let $T$ be a maximal torus with Lie algebra $t$. Let $\Sigma \subseteq \mathfrak{t}^{*}$ be the root system of $G$ with respect to $\mathfrak{t}$; fix an ordering of the roots with $\Sigma^{+}$the set of positive roots and $\Delta$ the set of simple roots. Then under the adjoint action of $T, \mathfrak{g}$ decomposes as an orthogonal direct sum

$$
\mathfrak{g}=\mathfrak{t} \oplus \sum_{\alpha \in \Sigma^{+}} \mathfrak{g}_{\alpha}
$$

where $\mathfrak{g}_{\alpha}$ is a two-dimensional subspace of $\mathfrak{g}$ such that for $X \in \mathfrak{t}, \operatorname{ad}(X)$ acts on $\mathfrak{g}_{\alpha}$ as

$$
\alpha(X)\left|\begin{array}{cc}
0 & -1 \\
1 & 0
\end{array}\right|
$$

with respect to some orthonormal basis of $\mathfrak{g}_{\alpha}$. For each $\alpha \in \Sigma$, let $k_{\alpha} \subseteq \mathfrak{t}$ denote the hyperplane

$$
k_{\alpha}=\{X \in \mathfrak{t} \mid \alpha(X)=0\} .
$$

Let $s_{\alpha}: \mathfrak{t} \rightarrow \mathfrak{t}$ denote reflection in the hyperplane $k_{\alpha}$ and let $W$, the Weyl group, be the finite group generated by the $s_{\alpha}, \alpha \in \Sigma$.

Let

$$
\mathfrak{t}_{+}=\left\{X \in \mathfrak{t} \mid \alpha(X) \geq 0 \forall \alpha \in \Sigma^{+}\right\} .
$$

Then $\mathfrak{t}_{+}$is a fundamental chamber for the action of $W$ so that each $X \in \mathfrak{t}$ is $W$-conjugate to exactly one element of $\mathfrak{t}_{+}$.

Now let $Z \in \mathfrak{g}$ and consider the orbit $O_{Z}$ of $Z$ under the adjoint action, i.e.,

$$
O_{Z}=\{\operatorname{Ad}(g) Z \mid g \in G\} .
$$

Then it is well known that $O_{Z} \cap \mathrm{t}$ is a finite set and in fact consists of exactly one $W$ orbit. It follows that every adjoint orbit $O_{Z}$ intersects $\mathfrak{t}_{+}$in a unique point.

Let

$$
V_{Z}=O_{Z} \cap \mathfrak{t}
$$

and let

$$
D_{Z}=\operatorname{conv}\left(V_{Z}\right) .
$$

Since the action of $G$ preserves the form $($,$) , all points of V_{Z}$ have the same norm and so are vertices of the polytope $D_{Z}$.

For $\alpha \in \Sigma^{+}$, denote the centralizer of $k_{\alpha}$ in $\mathfrak{g}$ by $\operatorname{cent}_{\mathfrak{g}}\left(k_{\alpha}\right)$. That is

$$
\operatorname{cent}_{\mathfrak{g}}\left(k_{\alpha}\right)=\left\{Z \in \mathfrak{g} \mid Z \cdot X=0 \forall X \in k_{\alpha}\right\}
$$

where we write $[Z, X]=Z \cdot X$. 
Lemma 2.1. $\operatorname{cent}_{\mathfrak{g}}\left(k_{\alpha}\right)=\mathfrak{t} \oplus \mathfrak{g}_{\alpha}$.

Proof. Let $Z \in \operatorname{cent}_{\mathfrak{g}}\left(k_{\alpha}\right)$ and write the decomposition of $Z$ according to (2.1) as

$$
Z=Z_{0}+\sum_{\beta \in \Sigma^{+}} Z_{\beta}
$$

with $Z_{0} \in \mathfrak{t}$ and $Z_{\beta} \in \mathfrak{g}_{\beta} \quad \forall \beta \in \Sigma^{+}$. Then

$$
\begin{aligned}
Z \cdot X & =0 \quad \forall X \in k_{\alpha} \\
& \Leftrightarrow \sum_{\beta \in \Sigma^{+}} X \cdot Z_{\beta}=0 \quad \forall X \in k_{\alpha} \\
& \Leftrightarrow \sum_{\beta \in \Sigma^{+}} \beta(X) Z_{\beta}^{\prime}=0 \quad \forall X \in k_{\alpha} \\
& \quad \text { where } Z_{\beta}^{\prime} \in \mathfrak{g}_{\beta} \text { is nonzero iff } Z_{\beta} \text { is nonzero } \\
& \Leftrightarrow Z_{\beta}=0 \quad \forall \beta \neq \alpha . \quad \square
\end{aligned}
$$

Denote the orthogonal complement in $\mathfrak{t}$ of $k_{\alpha}$ by $k_{\alpha}^{\perp}$. Then $\mathfrak{h}_{\alpha}=k_{\alpha}^{\perp} \oplus \mathfrak{g}_{\alpha}$ is a three-dimensional subalgebra of $\operatorname{cent}_{\mathfrak{g}}\left(k_{\alpha}\right)$ isomorphic to $\mathrm{su}(2)$, and we have the orthogonal decomposition

$$
\operatorname{cent}_{\mathfrak{g}}\left(k_{\alpha}\right)=k_{\alpha} \oplus \mathfrak{h}_{\alpha} .
$$

Define

$$
\mathfrak{m}_{\alpha}=\sum_{\substack{\beta \in \Sigma^{+} \\ \beta \neq \alpha}} \mathfrak{g}_{\beta} .
$$

Then

$$
\mathfrak{g}=k_{\alpha} \oplus \mathfrak{h}_{\alpha} \oplus \mathfrak{m}_{\alpha}
$$

is an orthogonal decomposition. This decomposition is preserved under the adjoint action of $\mathfrak{h}_{\alpha}$.

If $H_{\alpha}$ denotes the connected Lie subgroup of $G$ with Lie algebra $\mathfrak{h}_{\alpha}$ then by $\S 1$ for any $Z \in \mathfrak{h}_{\alpha}$, we may find $g \in H_{\alpha}$ such that $\operatorname{Ad}(g) Z=Z^{\prime} \in k_{\alpha}^{\perp}$. Furthermore, if the $k_{\alpha}^{\perp}$ component of $Z$ is nonzero, then we may arrange that the $k_{\alpha}^{\perp}$ component of $Z^{\prime}$ lies in the same Weyl chamber (i.e., half-line) as does that of $Z$. Applying the same reasoning to an arbitrary $Z \in \mathfrak{g}$ gives us the following.

Lemma 2.2. Let $\alpha \in \Sigma^{+}$and $Z \in \mathfrak{g}$. Then we can find $g \in H_{\alpha}$ such that if $\operatorname{Ad}(g) Z=Z^{\prime}$ then

(i) $Z^{\prime}$ has no $\mathfrak{g}_{\alpha}$ component;

(ii) the $k_{\alpha}$ components of $Z$ and $Z^{\prime}$ are identical;

(iii) the $\mathfrak{m}_{\alpha}$ components of $Z$ and $Z^{\prime}$ have the same norm;

(iv) the $k_{\alpha}^{\perp}$ components of $Z$ and $Z^{\prime}$ are in the same Weyl chamber of $k_{\alpha}^{\perp}$.

We will refer to the process described in the above lemma as "rotating $Z$ about the hyperplane $k_{\alpha}$ ". For $Z \in \mathfrak{g}$ and $\alpha \in \Sigma^{+}$, define the distance functions

$$
d_{\alpha}(Z)=\left|Z_{\alpha}\right|
$$


and

$$
d(Z)=\left|\sum_{\alpha \in \Sigma^{+}} Z_{\alpha}\right|=\left(\sum_{\alpha \in \Sigma^{+}} d_{\alpha}(Z)^{2}\right)^{1 / 2}
$$

The latter is just the distance from $Z$ to $\mathfrak{t}$. The basic algorithm can now be described. Let $Z \in \mathfrak{g}$, and set $Z^{1}=Z$. Construct a sequence $Z^{1}, Z^{2}, \ldots$ of elements of $\mathfrak{g}$ recursively as follows. Given $Z^{n-1}$, find $\alpha \in \Sigma^{+}$such that $d_{\alpha}\left(Z^{n-1}\right)$ is maximum. Use the formulae of $\S 1$ and Lemma 2.2 to find $g \in$ $H_{\alpha}$ such that $\operatorname{Ad}(g) Z^{n-1}=Z^{n}$ has no $\mathfrak{g}_{\alpha}$ component and satisfies the other conditions of the lemma. Then if $\left|\Sigma^{+}\right|=l$,

$$
\sum_{\beta \in \Sigma^{+}} d_{\beta}\left(Z^{n-1}\right)^{2} \leq l d_{\alpha}\left(Z^{n-1}\right)^{2}
$$

Thus,

$$
\begin{aligned}
d\left(Z^{n}\right)^{2} & =\sum_{\substack{\beta \in \Sigma^{+} \\
\beta \neq \alpha}} d_{\beta}\left(Z^{n}\right)^{2}=\sum_{\substack{\beta \in \Sigma^{+} \\
\beta \neq \alpha}} d_{\beta}\left(Z^{n-1}\right)^{2} \\
& =\sum_{\beta \in \Sigma^{+}} d_{\beta}\left(Z^{n-1}\right)^{2}-d_{\alpha}\left(Z^{n-1}\right)^{2} \\
& \leq\left(\frac{l-1}{l}\right) \sum_{\beta \in \Sigma^{+}} d_{\beta}\left(Z^{n-1}\right)^{2}=\left(\frac{l-1}{l}\right) d\left(Z^{n-1}\right)^{2}
\end{aligned}
$$

It follows that $d\left(Z^{n}\right) \rightarrow 0$ as $n \rightarrow \infty$. Set

$$
X^{n}=p\left(Z^{n}\right) \text {. }
$$

Then $X^{n-1}$ and $X^{n}$ differ only in the $k_{\alpha}^{\perp}$ direction and

$$
\left|X^{n}-X^{n-1}\right| \leq d_{\alpha}\left(Z^{n-1}\right)
$$

By (2.17) this becomes

$$
\left|X^{n}-X^{n-1}\right| \leq\left(\frac{l-1}{l}\right)^{(n-2) / 2} d\left(Z_{1}\right) .
$$

Thus $\left\{X^{n}\right\}$ is a Cauchy sequence and converges to an element $X^{\infty} \in \mathfrak{t}$ which is also the limit of the sequence $\left\{Z^{n}\right\}$. Since the orbit $O_{Z}$ is closed, $X^{\infty} \in O_{Z}$ so we have "diagonalized" $Z$ by performing an infinite series of rotations about hyperplanes. Furthermore

$$
\begin{aligned}
\left|X^{n}-X^{\infty}\right| & \leq \sum_{k=n}^{\infty}\left(\frac{l-1}{l}\right)^{(k-1) / 2} d\left(Z_{1}\right) \\
& =\frac{\left(\frac{l-1}{l}\right)^{(n-1) / 2}}{1-\left(\frac{l-1}{l}\right)^{1 / 2}} d\left(Z_{1}\right) \leq 2 l\left(\frac{l-1}{l}\right)^{(n-1) / 2} d\left(Z_{1}\right) .
\end{aligned}
$$

Each individual rotation is essentially a rotation in one of a finite number of $\mathrm{SU}(2)$ inside $G$. This is clearly an algorithm that could be implemented in a straightforward fashion on a computer. 
As an application, we use the algorithm to provide a new proof of a theorem of Kostant. We continue with the notation of the previous sections.

Theorem 3.1 (Kostant $[5])$. Let $X \in \mathfrak{t}$. Then $p\left(O_{X}\right)=D_{X}$.

Proof. Let $Z \in O_{X}$. Write $Z=Z^{1}$ and use the above algorithm to find a sequence $Z^{1}, Z^{2}, \ldots$ converging to $X^{\infty} \in \mathfrak{t}$ where the sequence of projections $p\left(Z^{n}\right)=X^{n}$ also converges to $X^{\infty}$. Note that $X^{\infty}$ must be $W$ conjugate to $X$. If we have rotated $Z^{n-1}$ about the hyperplane $k_{\alpha}$ to obtain $Z^{n}$, then as remarked in the previous discussion, $X^{n}$ differs from $X^{n-1}$ by an element of $k_{\alpha}^{\perp}$ and furthermore $X^{n}$ is further from the hyperplane $k_{\alpha}$ than $X^{n-1}$ is. Thus $X^{n-1}$ is between $X^{n}$ and $s_{\alpha}\left(X^{n}\right)$. It follows that $X^{n-1} \in D_{X^{n}}$ and so by $W$-invariance $D_{X^{n-1}} \subseteq D_{X^{n}}$. Therefore

$$
D_{X^{1}} \subseteq \cdots \subseteq D_{X^{n}} \subseteq \cdots \subseteq D_{X^{\infty}}
$$

and so

$$
X^{1} \in D_{X^{\infty}}=D_{X}
$$

Bû̃ $X^{1}=p(Z)$ and $Z \in O_{X}$ was arbitrary so that

$$
p\left(O_{X}\right) \subseteq D_{X}
$$

To show the reverse inclusion, suppose that $X \in \mathfrak{t}_{+}$and $Y \in D_{X} \cap \mathfrak{t}_{+}$. Consider a particle moving inside $t_{+}$which begins at $Y$ and always moves along a direction which is a positive multiple of a simple root. It is thus always moving perpendicularly away from one of the walls of $\mathfrak{t}_{+}$. Suppose whenever it has a choice (i.e., at the initial stage or whenever it reaches one of the walls of $t_{+}$) it chooses a direction in which it can move unimpeded in a straight line the longest. Clearly the particle would eventually approach infinity so in particular after a finite number of steps it will reach the boundary of $D_{X}$, say at a point

$$
Y^{\prime}=X-\sum_{\alpha \in \Sigma^{+}} r_{\alpha} \alpha
$$

where $r_{\alpha} \geq 0$. Here we have identified $\alpha$ with the unique element in $t$ such that

$$
\left.\alpha(X)=(\alpha, X) \quad \forall X \in \mathfrak{t} \text { (so that } \alpha \in k_{\alpha}^{\perp}\right) .
$$

Then clearly after another finite number of steps along simple root directions, the particle can reach $X$. Using the results of $\S 1$, we can choose $X=Z^{1}, Z^{2}$, $\ldots, Z^{k}=Z$ of $\mathfrak{g}$ such that $Z^{j+1}$ differs from $Z^{j}$ only by a rotation about the hyperplane $k_{\alpha_{j}}$, where $X^{j+1}$ differs from $X^{j}$ by a multiple of the simple root $\alpha_{j}$. Then $Z \in \mathfrak{g}$ is conjugate to $X$ and $p(Z)=Y$ as required.

\section{ACKNOWLEDGMENT}

The author would like to thank Michael Cowling for some useful remarks. 


\section{BIBLIOGRAPHY}

1. M. F. Atiyah, Convexity and commuting Hamiltonians, Bull. London Math. Soc. 14 (1982), $1-15$.

2. __, Angular momentum, convex polyhedra and algebraic geometry, Proc. Edinburgh Math. Soc. 26 (1983), 121-138.

3. V. Guillemin and S. Sternberg, Convexity properties of the moment mapping, Invent. Math. 67 (1982), 491-513.

4. G. J. Heckman, Projections of orbits and asymptotic behaviour of multiplicities for compact Lie groups, Invent. Math. 67 (1982), 333-356.

5. B. Kostant, On convexity, the Weyl group and the Iwasawa decomposition, Ann. Sci. Ecole Norm. Sup. 6 (1973), 413-455.

School of Mathematics, University of New South Wales, Kensington, New South Wales, 2033, Australia 